\title{
Quantification spatiale des précipitations. Applications au Nord-Est de la France
}

Spatial quantification of rainfall. Application to the Northeast of France

Räumliche Quantifizierung der Niederschläge. Anwendung auf den Nordosten

Frankreichs

Didier François et Joël Humbert

\section{(2) OpenEdition}

\section{Journals}

\section{Édition électronique}

URL : http://journals.openedition.org/rge/4159

DOI : $10.4000 /$ rge.4159

ISSN : 2108-6478

Éditeur

Association des géographes de l'Est

Édition imprimée

Date de publication : 1 janvier 2000

ISSN : 0035-3213

Référence électronique

Didier François et Joël Humbert, « Quantification spatiale des précipitations. Applications au Nord-Est de la France ", Revue Géographique de l'Est [En ligne], vol. 40 / 1-2 | 2000, mis en ligne le 03 août 2013, consulté le 08 septembre 2020. URL : http://journals.openedition.org/rge/4159; DOI : https://doi.org/ $10.4000 /$ rge.4159

Ce document a été généré automatiquement le 8 septembre 2020.

Tous droits réservés 


\title{
Quantification spatiale des précipitations. Applications au Nord-Est de la France
}

\author{
Spatial quantification of rainfall. Application to the Northeast of France \\ Räumliche Quantifizierung der Niederschläge. Anwendung auf den Nordosten \\ Frankreichs
}

Didier François et Joël Humbert

1 L'estimation des précipitations moyennes dans un bassin versant constitue le travail préalable à toute étude des relations pluie-débit. De sa précision dépend le calcul d'autres termes du bilan hydrologique: l'interception, l'évapotranspiration et l'infiltration. Les données uniquement issues des stations pluviométriques ne sont pas toujours suffisantes car la répartition spatiale des précipitations est le plus souvent liée à la topographie, à des paramètres morphométriques de l'environnement et de position sur les surfaces continentales. C'est pourquoi les méthodes classiques d'interpolation entre les postes qui ne tiennent pas compte des effets orographiques ne sont pas adaptées aux régions où le relief est très accidenté. Parfois simples et rapides à mettre en œuvre (polygones de Thiessen par exemple), ces méthodes ne peuvent donner des résultats satisfaisants qu'à l'échelle de grands bassins versants où les postes pluviométriques sont nombreux et répartis de manière homogène.

2 Les relations entre l'altitude et les précipitations ont déjà été largement étudiées (Schérer, 1977 ; Laborde 1984 ; Bénichou, Lebreton, 1987 ; Marand, Zumstein, 1990). Les Modèles Numériques de Terrain (MNT) permettent d'utiliser ces relations pour réaliser une véritable spatialisation des précipitations en tenant compte des effets du relief. Une méthode (PLUVIA) fondée sur ce type d'approche a été mise au point par le CEREG (Humbert, Perrin, 1993; Humbert, Perrin, Perron, 1993 et 1997; Humbert, 1995 ; Humbert, Drogue, Mahr, 1999). Elle diffère de la méthode AURELHY de Météo France (Bénichou, Lebreton, 1987) car les variables qui expliquent les précipitations sont issues directement de la grille d'un MNT et n'ont pas fait préalablement l'objet d'une Analyse 
en Composante Principale. Ceci permet d'identifier les caractéristiques topographiques qui déterminent la répartition des précipitations pour une année donnée, un mois, voire un épisode pluvieux.

Cette méthode a été appliquée conjointement par le CEREG et le CEGUM à deux secteurs distincts du Nord-Est de la France pour les précipitations annuelles de la période 1971-1990 (François, 1996 et 1997 ; Humbert, Mahr, Siefert, 1997). Ce travail a été réalisé dans le cadre d'une étude financée par l'Agence de l'Eau Rhin-Meuse pour déterminer des précipitations moyennes par zone de la codification hydrographique (d'une superficie moyenne de $30 \mathrm{~km} 2$ ).

\section{La méthode PLUVIA}

Chaque station pluviométrique est située à partir de coordonnées géographiques précises afin d'être placée sur la grille d'un MNT. Des paramètres relatifs aux altitudes environnantes des stations sont calculés en fonction de fenêtres d'extension croissante, centrées sur l'emplacement de la station ou excentrées selon 8 directions (nord, nordest, est, sud-est, sud, sud-ouest, ouest, nord-ouest, (fig. 1). La précision du MNT à utiliser et la taille maximale des fenêtres dépendent des caractéristiques topographiques de la région étudiée. Dans le cadre de ces travaux, des MNT à $100 \mathrm{~m}$ et à $1 \mathrm{~km}$ ont été utilisés respectivement sur une région de moyenne montagne (les Vosges) et sur une région de plateau (l'ouest de la Lorraine). Les fenêtres les plus grandes atteignent 30 et $32 \mathrm{~km}$.

Fig. 1 : Principe de construction des fenêtres utilisées pour le calcul des paramètres par station.

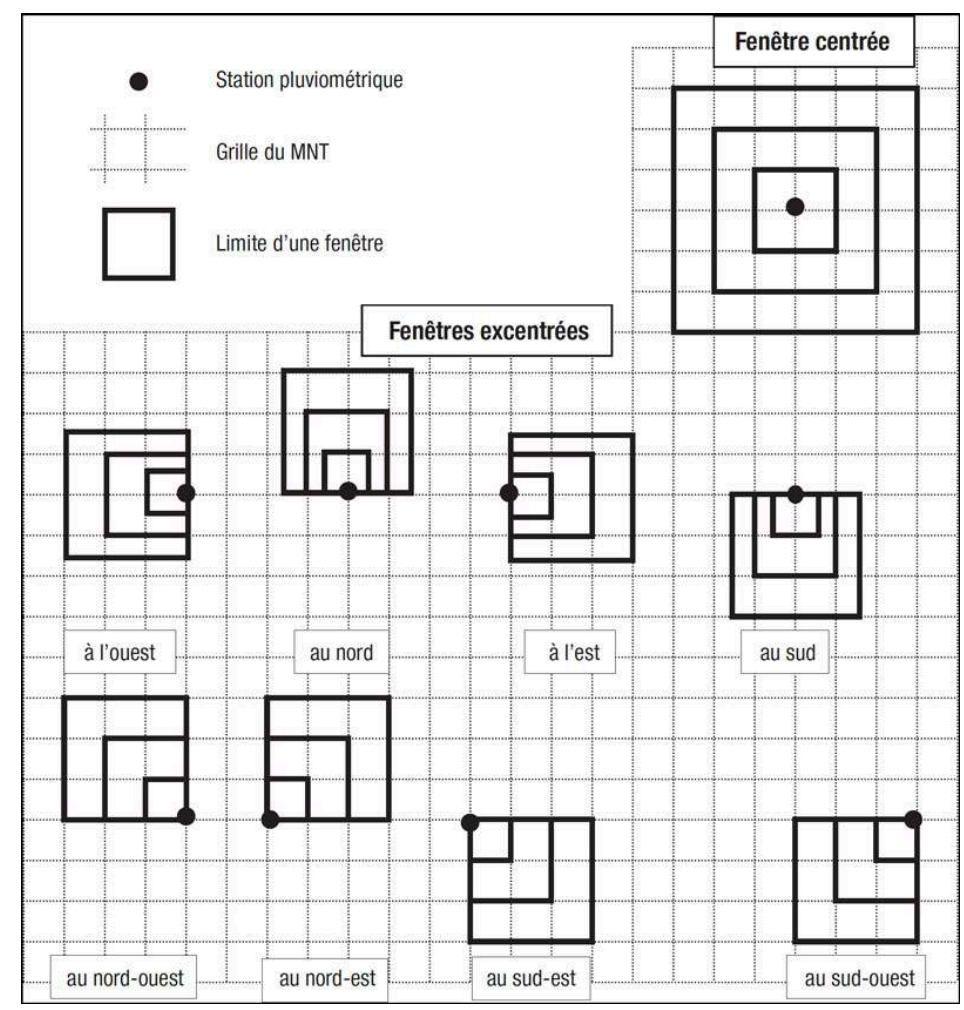

5 Toutes les altitudes situées à l'intérieur d'une fenêtre constituent une série de valeurs à partir desquelles des statistiques peuvent être calculées : moyenne, écart-type, mode, 
médiane, coefficient de variation, quantiles, etc. Un logiciel a d'ailleurs été développé spécifiquement par calculer toutes ces valeurs à partir de fichiers MNT (Mahr, Humbert, 1996). On obtient donc pour chaque station un nombre de paramètres qui dépend du nombre de direction des fenêtres, de l'incrément de croissance de la taille des fenêtres, de leur extension maximale et du nombre de variables statistiques calculées. Les coordonnées Lambert des stations s'ajoutent à ces paramètres. Ainsi, 2 160 paramètres ont été calculés pour le domaine Vosges-Alsace et 540 pour le domaine Lorraine ouest-Ardennes. Ceux-ci constituent les variables explicatives d'une régression multiple où la pluviométrie est la variable à expliquer.

Des variables calculées sur des fenêtres d'extension proche selon une même direction peuvent être très bien corrélées entre elles. Ces redondances ne sont pas gênantes pour la procédure de régression multiple pas à pas. Celle-ci permet de sélectionner la variable la plus pertinente pour un pas et un seuil de signification donnés, indépendamment d'un effet de corrélation avec une autre variable. Par contre l'utilisation d'autres procédures telle que best subset (détermination de la meilleure combinaison de prédicteurs) nécessite d'effectuer une sélection préalable des variables explicatives afin de minimiser la multicolinéarité.

En fonction du domaine étudié, les régressions sont réalisées avec les valeurs brutes ou leur logarithme. Pour une région montagneuse comme les Vosges où les altitudes et les précipitations sont très contrastées, les régressions en Log-Log fournissent de meilleurs résultats que les régressions arithmétiques. Les équations obtenues sont de la forme :

10 Où : $P$ est la hauteur de précipitation; $X 1, \ldots, X n$ sont les variables explicatives ; $a, \ldots, n$ sont les coefficients de régression ; $E$ est la constante de la régression

11 Les variables explicatives sélectionnées comme prédicteurs sont alors calculées pour tous les points d'une grille dont l'espacement dépend lui aussi de la région étudiée (1 $\mathrm{km}$ pour le domaine Vosges-Alsace et $2 \mathrm{~km}$ pour le domaine Lorraine ouest-Ardennes). L'équation appliquée à ces points définit une grille des précipitations calculées. Les résidus aux stations pluviométriques sont ensuite spatialisés par krigeage dans une grille identique à celle des précipitations calculées. La superposition de ces 2 grilles et la somme des valeurs par point aboutit à la carte des précipitations corrigées.

12 Ces valeurs (précipitations calculées par les régressions puis corrigées par les résidus) sont comparées avec les précipitations de postes qui n'ont pas été inclus dans les calculs. Cette phase de validation rend compte du plus ou moins bon ajustement du modèle à la situation pluviométrique étudiée. Un écart important entre les précipitations mesurées et les précipitations calculées à un poste de validation justifie un retour à la phase de régression et au choix d'autres prédicteurs.

\section{Application de la méthode}

13 La méthode a été appliquée aux précipitations moyennes interannuelles et aux précipitations annuelles de la période 1971-1990. Compte tenu de l'étendue de la zone étudiée $(58000 \mathrm{~km} 2)$ et de la variété des types de relief (relief de côtes, massif vosgien et ardennais, plaine d'Alsace), une étude préalable a défini des régions homogènes où un seul modèle pouvait être mis en œuvre (François, Gille, Zumstein, 1995). Des 
corrélations simples "précipitations - altitudes lissées » ont conduit à différencier plusieurs zones à l'intérieur desquelles les coefficients de corrélation sont élevés. Une limite majeure, de direction nord est - sud-ouest, a été tracée au milieu de la zone étudiée (fig. 2). Elle sépare le domaine occidental, caractérisé par le relief de côtes et le Massif Ardennais, du domaine oriental composé du Massif Vosgien et de la Plaine d'Alsace. Ces domaines ont été étudiés séparément par les deux laboratoires avec des outils et des méthodes sensiblement différentes, le domaine occidental par le CEGUM et le domaine oriental par le CEREG. Une aire de chevauchement de $50 \mathrm{~km}$ de large a été définie le long de la limite afin de vérifier la convergence des modèles élaborés par les deux laboratoires.

Fig. 2 : Quantification Spatiale des Précipitations. Région étudiée.

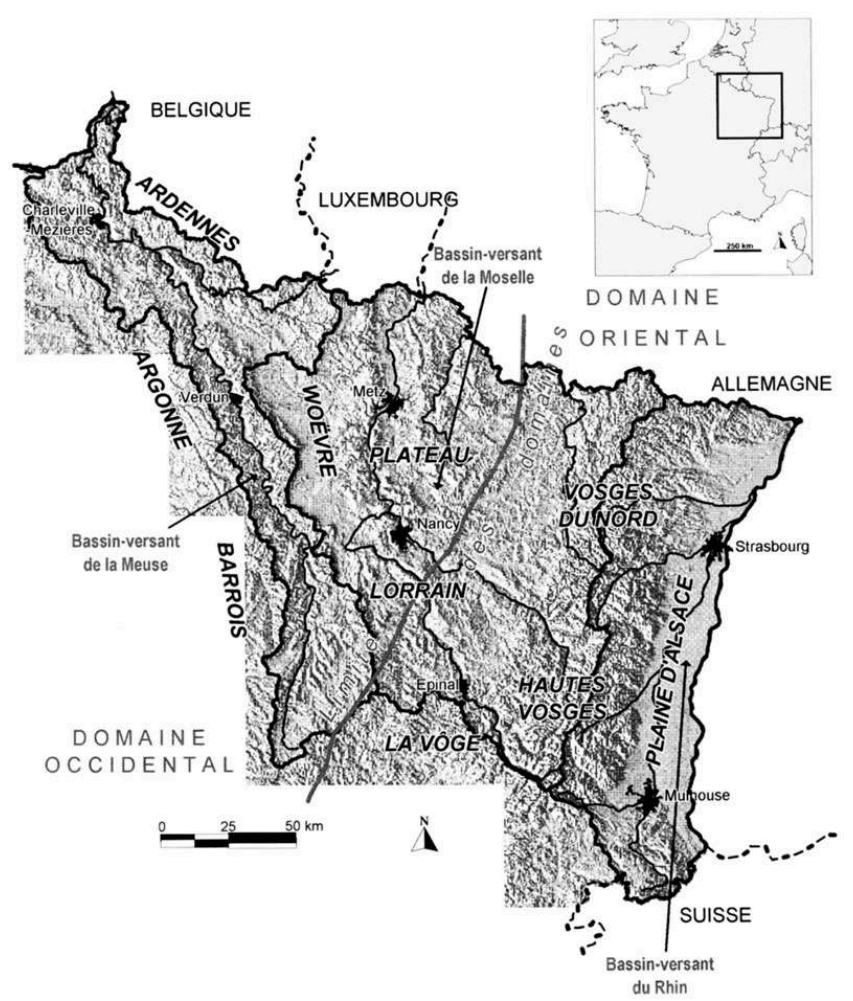

\section{A. Domaine oriental : Massif vosgien-Alsace}

La partie orientale de la zone d'étude s'étale approximativement entre la longitude de Vittel et le Rhin, mais intègre aussi les contreforts septentrionaux du Jura au sud-est, ainsi que la retombée méridionale des Vosges. Sur cette surface de l'ordre de 28000 km2 apparaissent des paysages relativement contrastés : Plateau Lorrain, Massif des Hautes Vosges, Vosges du Nord, Plaine d'Alsace, collines du Sundgau... Cette mosaïque topo-morphométrique se traduit également par une diversité des caractéristiques pluviométriques, induites notamment par le fait que les flux perturbés d'ouest ou de sud-ouest (les plus fréquents, Paul 1992) abordent la barrière vosgienne de façon plus ou moins perpendiculaire. Il s'en suit un puissant effet orographique générant des contrastes marqués de part et d'autre de la ligne de crête vosgienne. 
Pour tenter de mieux cerner cette hétérogénéité dans une première étape, en considérant la période moyenne 1971-1990, le modèle Pluvia a été régionalisé et appliqué sur chacune des zones précédemment citées. Bien que très satisfaisante au plan des résultats, cette approche a été abandonnée du fait de sa lourdeur et des problèmes liés à la jonction des isohyètes lors de l'étape de la cartographie relative à l'ensemble de la région étudiée. Chaque année a donc été traitée en appliquant une seule régression sur l'ensemble du domaine oriental (Humbert, Mahr, Siefert, 1997). Il se trouve que, malgré cette simplification, la combinaison de quelques prédicteurs suffit à prendre en compte la différenciation spatiale des effets orographiques, pourvu que les stations utilisées soient suffisamment représentatives, ce qui est le cas ici. Le nombre de stations utilisées pour le calcul des régressions annuelles varie entre 167 et 183 ; un lot de 12 à 20 postes a été réservé chaque année à la validation, en prenant soin d'utiliser des jeux différents d'une année à l'autre et de s'assurer de leur représentativité spatiale. Le nombre de postes de validation peut paraître faible, mais différents essais réalisés récemment ont montré que si l'on augmente leur nombre (60 à près de 100 postes, et en diminuant donc d'autant les postes de calcul), les résultats ne sont guère affectés.

L'ensemble des équations retenues, ainsi que leurs principales caractéristiques sont rassemblées dans le tableau 1 . Chaque régression est significative au seuil de $1 \%$. On constate que les coefficients de détermination sont relativement élevés, s'échelonnant entre 0,85 et 0,92 . Certaines années s'avèrent relativement difficiles à modéliser, comme par exemple 1971 ou 1985 ; d'autres paraissent plus facilement accessibles, telles les années 1977 et 1979, ou encore la moyenne 1971-90.

Tableau 1 : Récapitulatif des régressions utilisées sur le domaine oriental

\begin{tabular}{|c|c|c|c|c|c|c|c|c|}
\hline Année & Equation & Test $\mathrm{F}$ & 12 & $\mathrm{p}$ & ECR & ECE & n & $\mathrm{n}$ valid. \\
\hline 1971 & $2,03+0,403$ MSE22 - 0,449ME22 + 0,438MNE15 - 0,49PDSW30 - 0,096PDE30 + 0,476DC8 & 153,9 & 0,852 & 690 & 80,2 & 11,2 & 167 & 14 \\
\hline 1972 & $1,913+0,3$ MSW8 + 0,078ENE30 + 0,275PDSW2 - 0,609PDSW30 + 0,359PDN 16 & 276,7 & 0,895 & 879,2 & 88,2 & 10,3 & 169 & 13 \\
\hline 1973 & $1,897+0,28 \mathrm{MC} 3-0,439 \mathrm{MSW} 30+0,106 \mathrm{ESW} 30+0,031$ EE3 $3+0,083$ ENE30 $+0,421$ PQC17 & 273,4 & 0,909 & 915,5 & 93,2 & 10,3 & 171 & 13 \\
\hline 1974 & $1,846+0,235 \mathrm{MC} 2+0,563 \mathrm{MW7}-0,325 \mathrm{MW} 30+0,045 E S E 14-0,558 \mathrm{PDSW} 30+0,514 \mathrm{PDNN} 30$ & 308,9 & 0,918 & 1076 & 121,6 & 11,5 & 173 & 12 \\
\hline 1975 & $1,713+0,206 \mathrm{MNE} 2+0,118 E E 30-0,281$ PDSW30 + 0,301PDN30 + 0,161DDSW2 & 242,4 & 0,878 & 895,6 & 97,6 & 10,7 & 174 & 16 \\
\hline 1976 & $1,931+0,281 \mathrm{MC} 14+0,329 \mathrm{MS} 2+0,101$ EE30 $-0,598$ PDSW $30+0,252$ PDN14 & 252,8 & 0,887 & 750 & 83,3 & 11,5 & 167 & 18 \\
\hline 1977 & $1,334-0,509$ PQSW30 $+0,233$ PQS30 $+0,346$ PQN30 $+0,135$ DDE30 $+0,462$ MW 8 & 349,3 & 0,914 & 1171 & 119,4 & 10,6 & 171 & 15 \\
\hline 1978 & $2,063-0,795 \mathrm{MSW} 30+0,284 \mathrm{MS} 13+0,177$ ESW $30+0,442$ PDN $13+0,329$ DQSW2 & 287,6 & 0,898 & 1089 & 119,7 & 11,8 & 170 & 18 \\
\hline 1979 & $2,002+0,404 \mathrm{MW} 8-0,544 \mathrm{MSW} 30+0,117 \mathrm{MNE} 8+0,09 \mathrm{CSW} 22+0,483 \mathrm{PDC} 15$ & 394,5 & 0,92 & 1199,6 & 130,4 & 9,6 & 177 & 14 \\
\hline 1980 & $2,292+0,454 \mathrm{MW} 8-0,666 \mathrm{MW} 30+0,111 \mathrm{EW} 23+0,684 \mathrm{PQC} 15-0,473 \mathrm{PQSW} 30+0,206 \mathrm{DQN} 30$ & 248,6 & 0,908 & 1097,6 & 127 & 11,5 & 182 & 14 \\
\hline 1981 & $1,991-0,55 \mathrm{MSW} 30+0,053 \mathrm{ESE} 22+0,135 \mathrm{CW} 13+0,629 \mathrm{PDW} 7+0,4 \mathrm{PDN} 22$ & 320,9 & 0,902 & 1307,1 & 144,2 & 11,9 & 181 & 14 \\
\hline 1982 & $2,11-0,681 \mathrm{MSW} 30+0,135 \mathrm{ESW} 30+0,405 \mathrm{PDN} 8+0,577 \mathrm{DC} 22$ & 336,1 & 0,883 & 1264,3 & 154,8 & 12 & 183 & 15 \\
\hline 1983 & $1,972+0,426$ MW5 $-0,597 \mathrm{MSW} 30+0,099$ ESW $25+0,042$ EE25 $+0,321$ PQC15 $+0,183$ PQNE15 & 285,2 & 0,909 & 1194,7 & 141,5 & 11,2 & 178 & 20 \\
\hline 1984 & $2,085+0,286 \mathrm{MW} 8-0,693 \mathrm{MSW} 30+0,086 \mathrm{ESW} 23+0,172$ PDNE $15+0,55 \mathrm{DC} 15$ & 300,4 & 0,895 & 1074,5 & 127,7 & 11,6 & 182 & 17 \\
\hline 1985 & $2,022+0,067$ ESW $3+0,071$ EE $30+0,793$ PQC10 $-0,492$ PQSW30 $-0,248$ PQE3 $+0,21$ PQN30 & 181,4 & 0,863 & 873,9 & 113,8 & 13 & 180 & 15 \\
\hline 1986 & $1,892+0,624$ MC9 $-0,872$ MSW30 $-0,04$ ENE9 + 0,317PDN16 + 0,424DDSW23 & 258,9 & 0,883 & 1282,6 & 155,8 & 12,2 & 177 & 12 \\
\hline 1987 & $2,309+0,062$ ESW $3+0,161$ CE $30+0,598$ PDC $9+0,268$ PDC $30-0,571$ PDSW 30 & 221,3 & 0,864 & 1152,8 & 128,4 & 11,3 & 180 & 15 \\
\hline 1988 & $1,999+0,564 \mathrm{MW} 8-0,796 \mathrm{MSW} 30+0,149 \mathrm{ESW} 30+0,197 \mathrm{PQS} 30+0,362$ PQN 15 & 243,8 & 0,878 & 1247 & 160 & 13 & 175 & 13 \\
\hline 1989 & $2,238+0,417 \mathrm{MC} 15+0,187 \mathrm{MSW} 2-0,448$ PQSW30 $-0,172$ PQSE $30+0,283$ PQNE15 & 236,4 & 0,879 & 928,9 & 101,9 & 10,4 & 169 & 15 \\
\hline 1990 & $1,904+0,853 \mathrm{MW} 9-0,453 \mathrm{MW} 30+0,078 \mathrm{CSE} 23-0,053 \mathrm{CE} 9-0,448 \mathrm{PDSW} 30+0,486 \mathrm{PDN} 30$ & 194,3 & 0,877 & 1067 & 138 & 12,7 & 170 & 14 \\
\hline $1971-90$ & $1,933+0,057$ EE21+0,207PQW3 $-0,498$ PQSW29 $+0,174$ PQN29+0,492DC15 & 372,7 & 0,923 & 1065,8 & 103,3 & & 161 & \\
\hline & 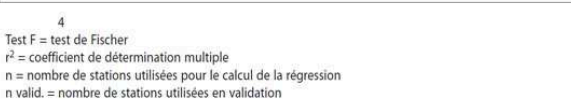 & \multicolumn{7}{|c|}{$\begin{array}{l}P=\text { précipitations moyennes mesurees }(\mathrm{mm}) \\
E C R=\text { écrt-type des résidus }(\mathrm{mm}) \\
\mathrm{ECE}=\text { écart-type de léreur }(\%)\end{array}$} \\
\hline & \multicolumn{8}{|c|}{ 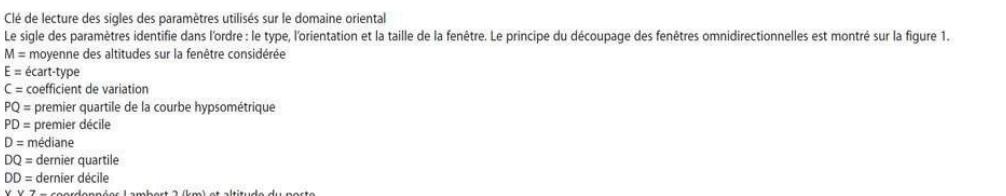 } \\
\hline
\end{tabular}


Le nuage complet des 310 points de validation retenus (toutes périodes confondues) est représenté sur la figure 3 . On peut noter que $75 \%$ des erreurs sont inférieures à $\pm 10 \%$, avec un écart-type de $9.3 \%$; quant à l'erreur maximale, elle est de l'ordre de $33 \%$. Une légère tendance à la surestimation apparait toutefois au-delà de $1800-2000 \mathrm{~mm}$. Cette surestimation est essentiellement imputable à quelques stations du versant alsacien des Hautes Vosges (Sewen-MF, Mollau, Rimbach-près-Masevaux) pour lesquelles la reconstitution semble souvent difficile. Indépendamment des incertitudes de mesure, des effets de site ou d'abri microclimatique non pris en compte par le modèle, ne sont pas à exclure.

Fig. 3 : Validation sur l'ensemble des périodes étudiées (Domaine oriental)

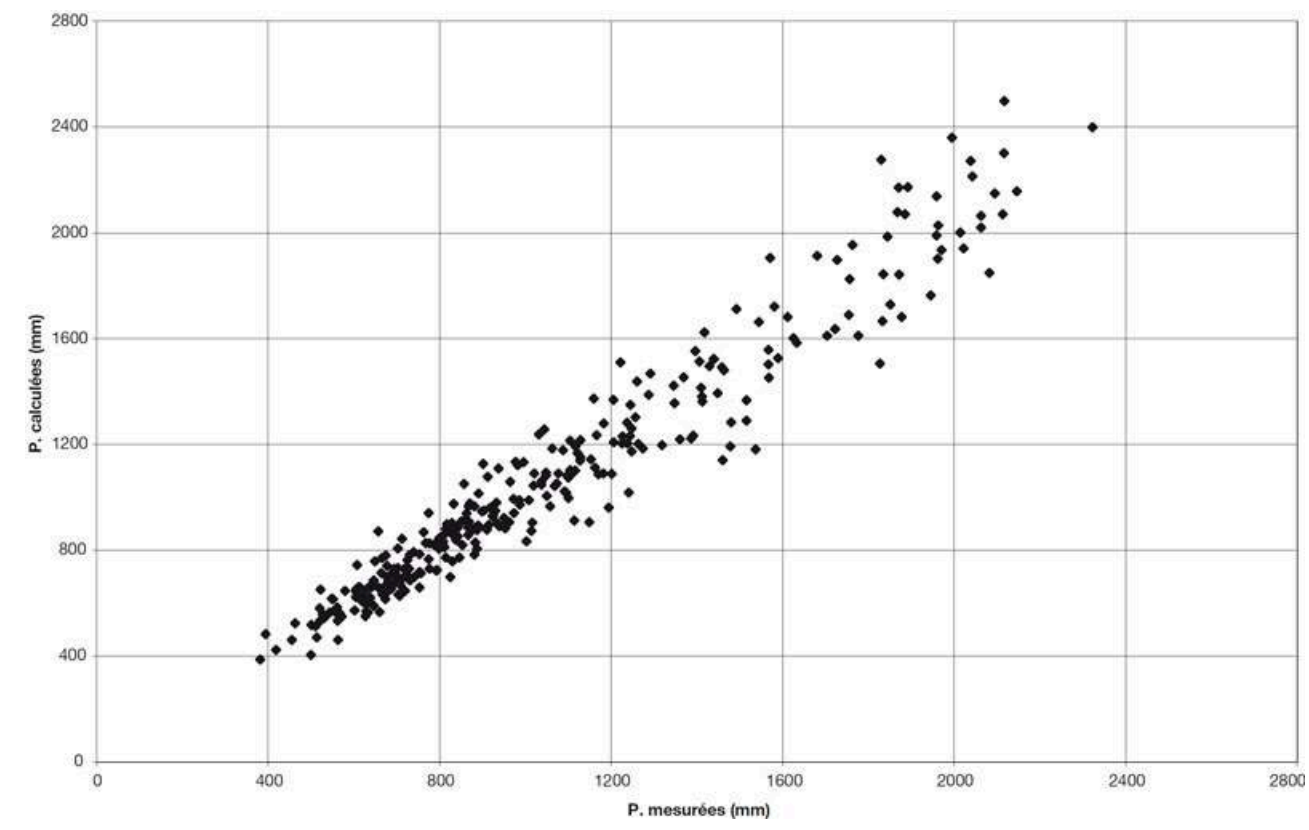

En règle générale, quatre à six prédicteurs suffisent à expliquer près de $90 \%$ de la variance des précipitations brutes, en moyenne pour l'ensemble des régressions calculées. Chaque année possède évidemment son lot de prédicteurs propres, en fonction de la statistique des types de temps pluvieux qui ont caractérisé la période. La confrontation prédicteurs-types de temps est actuellement en cours d'analyse, notamment sur des pas de temps plus fins. Mais néanmoins, dans le cas présent, on constate qu'au niveau annuel s'instaure une logique assez bien résumée par l'examen de la régression établie pour la moyenne interannuelle 1971-1990. Les prédicteurs sont expliqués ci-après dans un ordre hiérarchique, en fonction du poids de leur coefficient standardisé (du plus fort au plus faible). On pourra se reporter au tableau 1 pour l'explication de la dénomination des variables explicatives.

- La fenêtre DC15 dotée d'un signe positif révèle un effet d'ascendance classique des masses d'air, lié à la masse montagneuse centrée sur le point de mesure.

- La présence du prédicteur PQSW29, associé à un signe négatif, exprime l'effet d'abri dont bénéficient les points placés dans l'ombre pluviométrique d'une masse montagneuse plus ou moins élevée, s'étalant au SW. Systématiquement présente avec une fenêtre de grande taille, cette variable traduit aussi la prédominance des types de temps perturbés de secteur SW ou WSW. 

surcroît de précipitations en ce point. Ceci doit être à nouveau mis en relation avec la fréquence et la trajectoire des flux perturbés de SW, ainsi que leur potentiel pluviogène.

23 - Enfin, avec un poids moindre, apparaît un écart-type d'altitude (EE21), signalant que plus la variabilité des altitudes est élevée à l'est du point, plus les précipitations sont abondantes. La présence d'un écart-type est fréquente; elle rappelle que l'hétérogénéité du relief peut être une cause de surcroît pluviométrique, notamment en cas de situations convectives.

24 - Ainsi, cet examen sommaire montre qu'il existe un lien étroit entre la configuration topographique de la zone étudiée, la distribution spatiale des précipitations et la statistique des types de temps intéressant le Nord-Est français. Une logique du même type a pu être constatée sur le domaine occidental, bien que les paysages lorrains ou ardennais aient peu de points communs avec la zone orientale. La paramétrisation croissante et omnidirectionnelle du relief permet, dans une certaine mesure, d'optimiser l'analyse de la répartition spatiale des précipitations, en combinant des prédicteurs topo-morphométriques variés, orientés, tout en respectant le poids des facteurs locaux et celui des paramètres d'envergure plus régionale.

\section{B. Domaine occidental : Plateau lorrain-Ardennes}

Les reliefs du domaine occidental constituent la première barrière orographique de l'est du Bassin Parisien. Principalement orientés nord-sud perpendiculairement aux flux dominants d'ouest, ils se caractérisent par des altitudes maximales s'échelonnant, du nord au sud, de $400 \mathrm{~m}$ sur le plateau de Langres et du Barrois à $300 \mathrm{~m}$ dans l'Argonne pour atteindre $500 \mathrm{~m}$ dans les Ardennes. Ils dominent la Champagne dont les altitudes dépassent rarement $250 \mathrm{~m}$.

26 À l'est, les côtes de Meuse et de Moselle atteignent régulièrement 300-350 m et encadrent la plaine de la Woëvre où les altitudes sont inférieures à $200 \mathrm{~m}$. La limite orientale du domaine correspond à une région de collines au nord-est et de plateau au sud-ouest dont les altitudes dépassent rarement $300 \mathrm{~m}$.

134 postes pluviométriques se répartissent dans cette région de $30000 \mathrm{~km} 2$. Ils sont représentatifs d'altitudes et de pluviosités relativement variées. Il existe des mesures pour les régions les plus élevées et les plus arrosées comme pour les régions les plus basses et les plus sèches.

28 La banque de données topographiques est issue d'un MNT de $1 \mathrm{~km}$ d'espacement. Compte tenu de la lourdeur des calculs avec un nombre important de variables, celle-ci a été considérablement simplifiée au cours de l'étude.

Une première simplification de la banque a été réalisée en adoptant une progression géométrique de la taille des fenêtres $(2,4,8,16$ et $32 \mathrm{~km})$. Ainsi pour une fenêtre donnée, la surface de la fenêtre précédente ne représente que $25 \%$ de sa surface, ce qui limite les corrélations élevées entre les variables. D’autre part, les régressions réalisées avec les précipitations des années particulières montrent que les coefficients de

Revue Géographique de l'Est, vol. 40 / 1-2 | 2000 
détermination (R2) et les écarts-types résiduels sont comparables si on utilise des fenêtres d'une extension maximum de 32 ou de $16 \mathrm{~km}$. Par contre en réduisant leur taille maximum à $8 \mathrm{~km}$, les valeurs de R2 diminuent de manière significative. Les fenêtres de $32 \mathrm{~km}$ d'extension ont donc été abandonnées. À l'issue de toutes ces vérifications, une banque de données topographiques de 75 variables a été constituée et utilisée pour établir les régressions à l'échelle interannuelle.

Les premiers essais de régressions multiples pas à pas réalisés pour des années particulières montrent que certaines variables ne sont jamais utilisées, mais 20 d'entre elles apparaissent plusieurs fois comme prédicteurs. Elles constituent donc une sélection des variables les plus pertinentes pour expliquer la répartition des précipitations annuelles. Cette matrice limitée à 20 variables a été utilisée pour réaliser la spatialisation des précipitations annuelles de 1971 à 1990 (tableau 2). Ce choix limite les calculs pour lesquels une autre procédure de régression (Best Subsets Regression) a été mise en œuvre. Celle-ci envisage toutes les combinaisons de prédicteurs pour en sélectionner la plus satisfaisante.

Tableau 2 : Paramètres des régressions (domaine occidental)

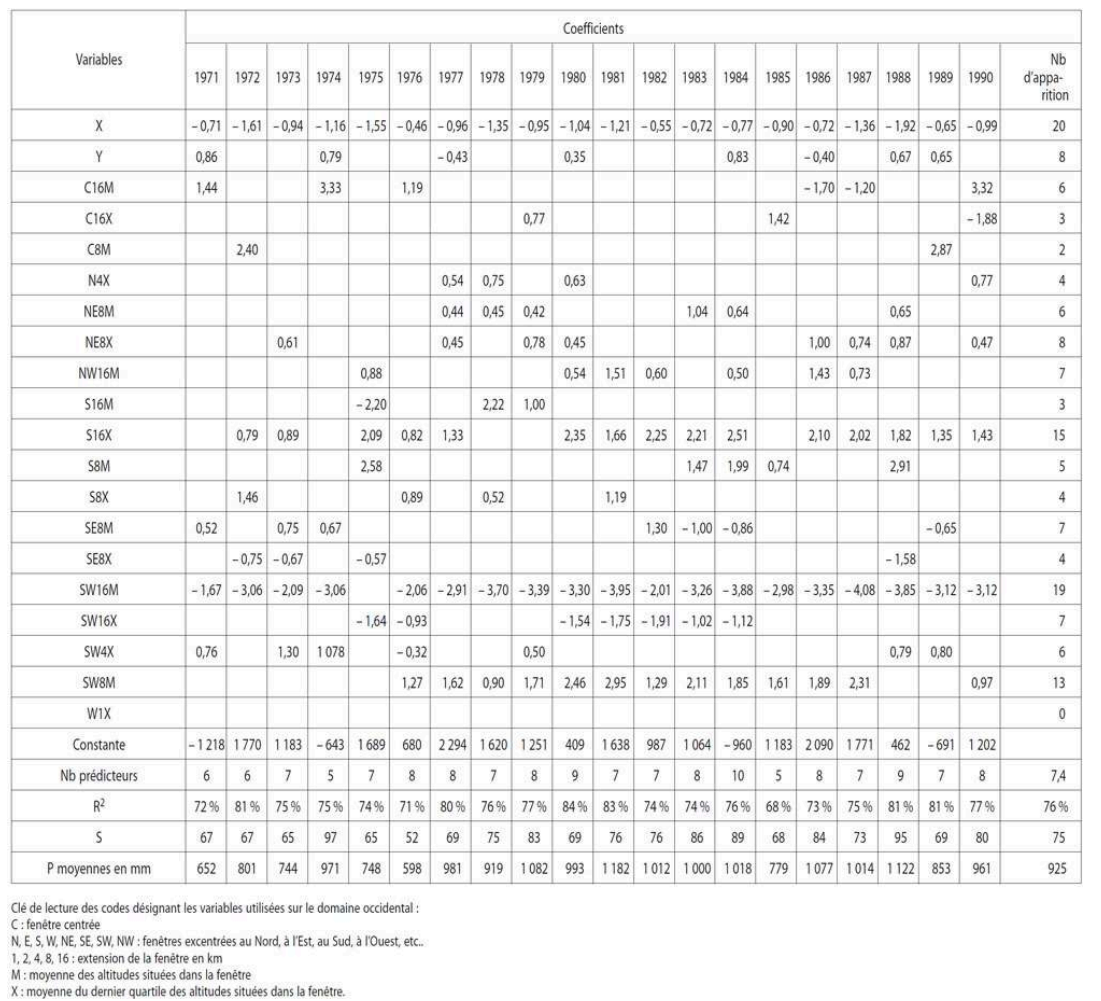

Les précipitations annuelles de la période 1971-1990 ont été spatialisées à l'aide de l'équation suivante :

$P=1536-1,2 \times X+3,09 \times C 8 M-1,78 \times S W 16 M$

33 Le lot de prédicteurs se compose du X Lambert, de l'altitude moyenne de la fenêtre centrée de $8 \mathrm{~km}$ d'extension et de l'altitude moyenne d'une fenêtre excentrée au sudouest de $16 \mathrm{~km}$ d'extension. Ils expliquent $80 \%$ de la variance, l'écart-type résiduel étant de $65 \mathrm{~mm}$. 
La validation de ce modèle s'est révélée satisfaisante car les écarts maximums entre les précipitations mesurées et les précipitations calculées puis corrigées, ne dépassent pas $10 \%$.

Pour les années particulières, les précipitations annuelles s'échelonnent de $361 \mathrm{~mm}$ en 1971 à Essey-et-Maizerais dans la Woëvre à 1686 mm en 1981 à Bouillon dans les Ardennes. Les résultats sont assez contrastés. Les coefficients de détermination sont compris entre 68 et $84 \%$ (76\% en moyenne). L'écart-type résiduel varie de 52 à $97 \mathrm{~mm}$ (75 mm en moyenne) selon le caractère plus ou moins humide de l'année. Plus de 7 prédicteurs ont été retenus en moyenne.

Parmi les 20 variables sélectionnées initialement (tableau 2), 4 apparaissent très souvent dans les régressions :

- Le X Lambert est présent dans chaque régression avec un coefficient négatif qui rend compte de la diminution longitudinale des précipitations.

- La moyenne des altitudes d'une fenêtre de $16 \mathrm{~km}$ excentrée au sud-ouest (SW16M) a été sélectionnée 19 années sur 20. Ses coefficients sont toujours négatifs (comme à l'échelle interannuelle) et semblent liés à un effet d'abri par rapport aux reliefs situés au sud-ouest.

9 - La moyenne des altitudes maximales d'une fenêtre de $16 \mathrm{~km}$ excentrée au sud (S16X), retenue pour 15 années, a un coefficient positif révélateur d'un effet d'ascendance qui provoque des précipitations orographiques.

- La moyenne des altitudes d'une fenêtre de $8 \mathrm{~km}$ excentrée au sud-ouest (SW8M) apparaît pour 13 années. Contrairement à la même fenêtre de $16 \mathrm{~km}$ d'extension, ses coefficients sont tous positifs et traduisent un effet d'ascendance des masses d'air.

1 Les autres variables sont utilisées moins souvent et leur contribution dans les régressions reste beaucoup plus faible.

La phase de validation confirme les résultats globalement satisfaisants, obtenus par les régressions. L'écart moyen en valeur absolue entre les précipitations issues du modèle et les valeurs réellement mesurées aux postes de validation est de $7 \%$, avec un écart maximum de $32 \%$.

III. Cartographie et comparaison avec la méthode du krigeage

Parmi les nombreuses cartes réalisées sur les deux domaines d'étude, nous présentons ici la cartographie des précipitations moyennes interannuelles, relatives à la période 1971-1990 et couvrant l'intégralité de la région. En effet, bien que les deux domaines aient été étudiés par des laboratoires distincts avec des outils et des régressions différentes, les résultats convergent dans la zone tampon qui les borde. La figure 4a résulte donc de la juxtaposition des grilles issues des deux domaines. Par ailleurs, compte tenu des nombreuses validations déjà appliquées à l'échelle annuelle sur cette période et du caractère satisfaisant des résultats, cette cartographie est fondée sur l'intégralité des postes disponibles et n'est donc pas validée. Au total, 161 stations ont été utilisées sur le domaine oriental et 132 sur le domaine occidental.

Fig. 4 : Quantification Spatiale des Précipitations. Période de référence 1971-1990 
4a : Précipitations moyennes annuelles spatialisées par la méthode PLUVIA

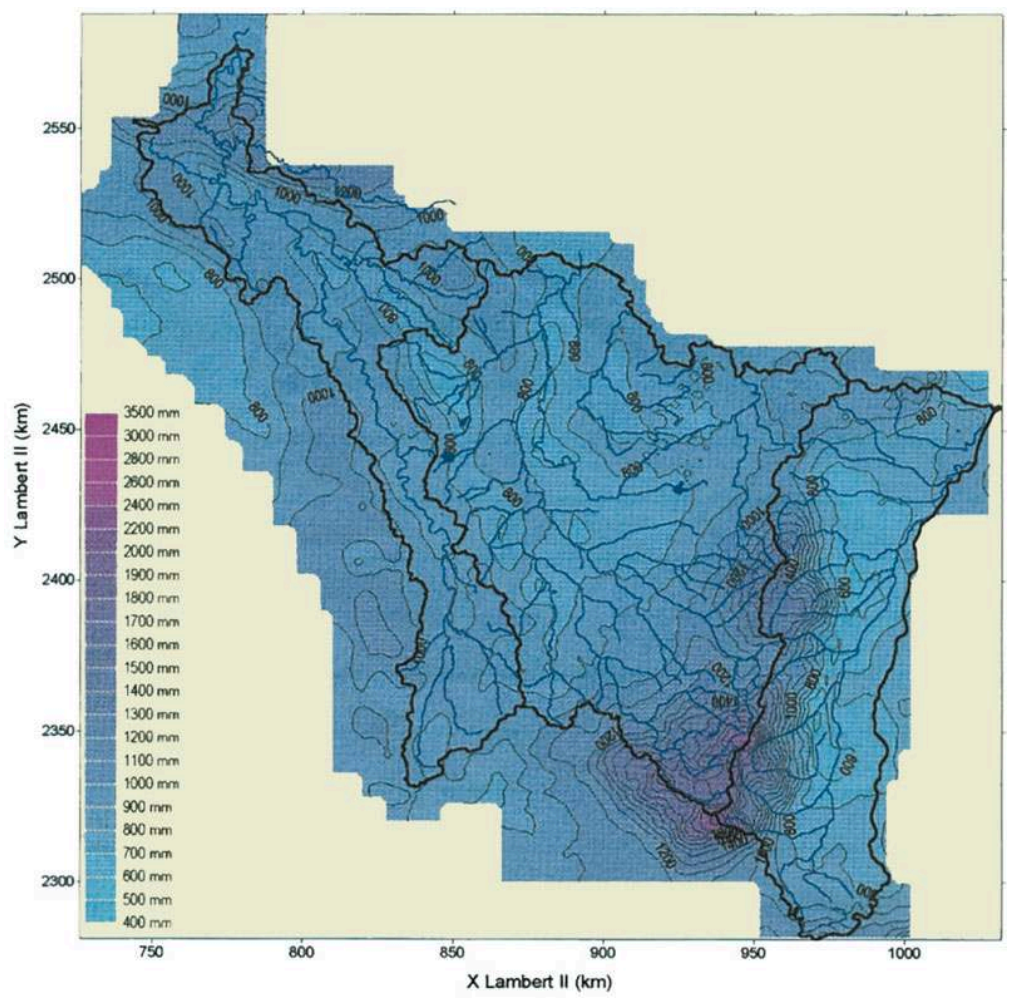

Fig. 4b : Précipitations moyennes annuelles spatialisées par krigeage

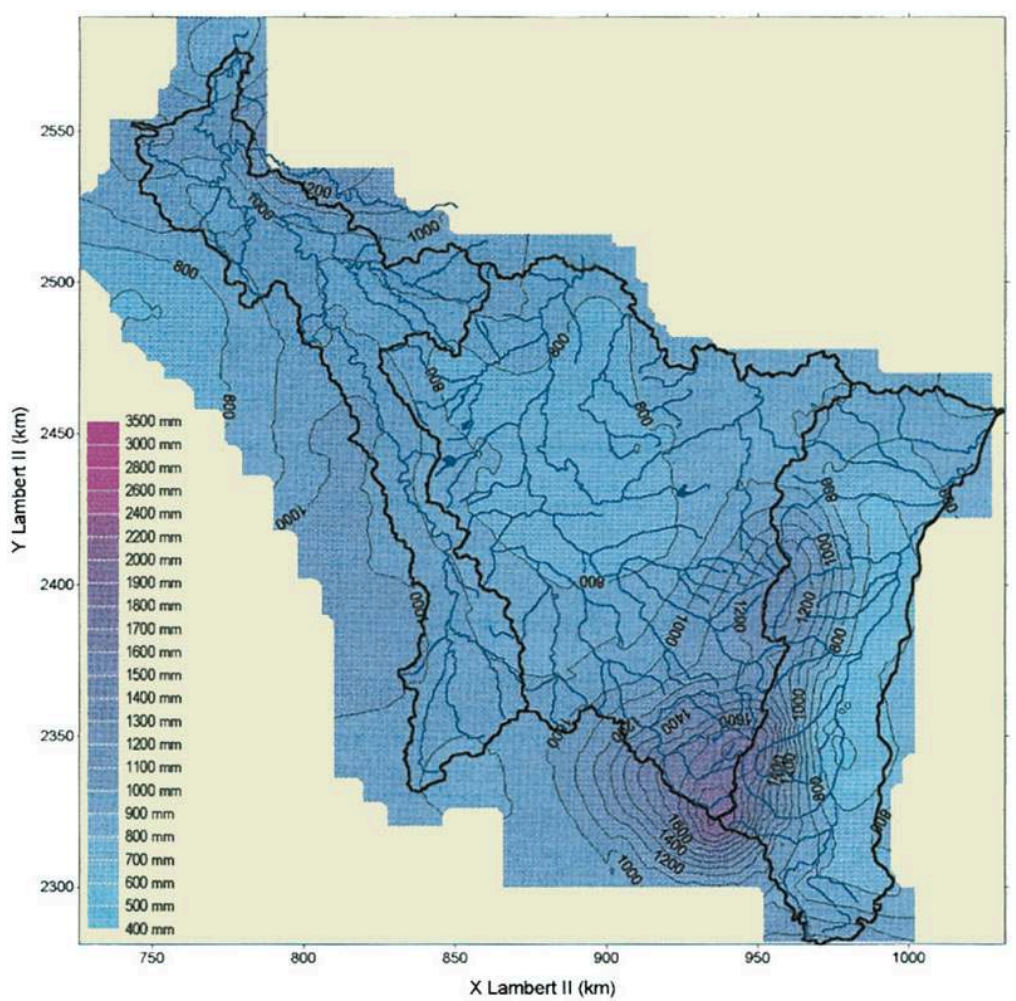

45 Cette carte issue de la méthode PLUVIA a été comparée à un tracé d'isohyètes réalisé par un krigeage simple (figure $4 \mathrm{~b}$ ), à partir d'un réseau de mesures identique 
(Delhomme, 1978; Obled, 1987). Le variogramme expérimental, ajusté à un modèle gaussien (Englund, Sparks, 1991), est représenté sur la figure 5. Compte tenu de l'étendue de la région étudiée, les deux cartes ne sont pas fondamentalement différentes. Le réseau pluviométrique possède une densité suffisante pour que les isohyètes suivent les principaux reliefs.

Fig. 5 : Variogramme des précipitations moyennes annuelles (1971-1990)

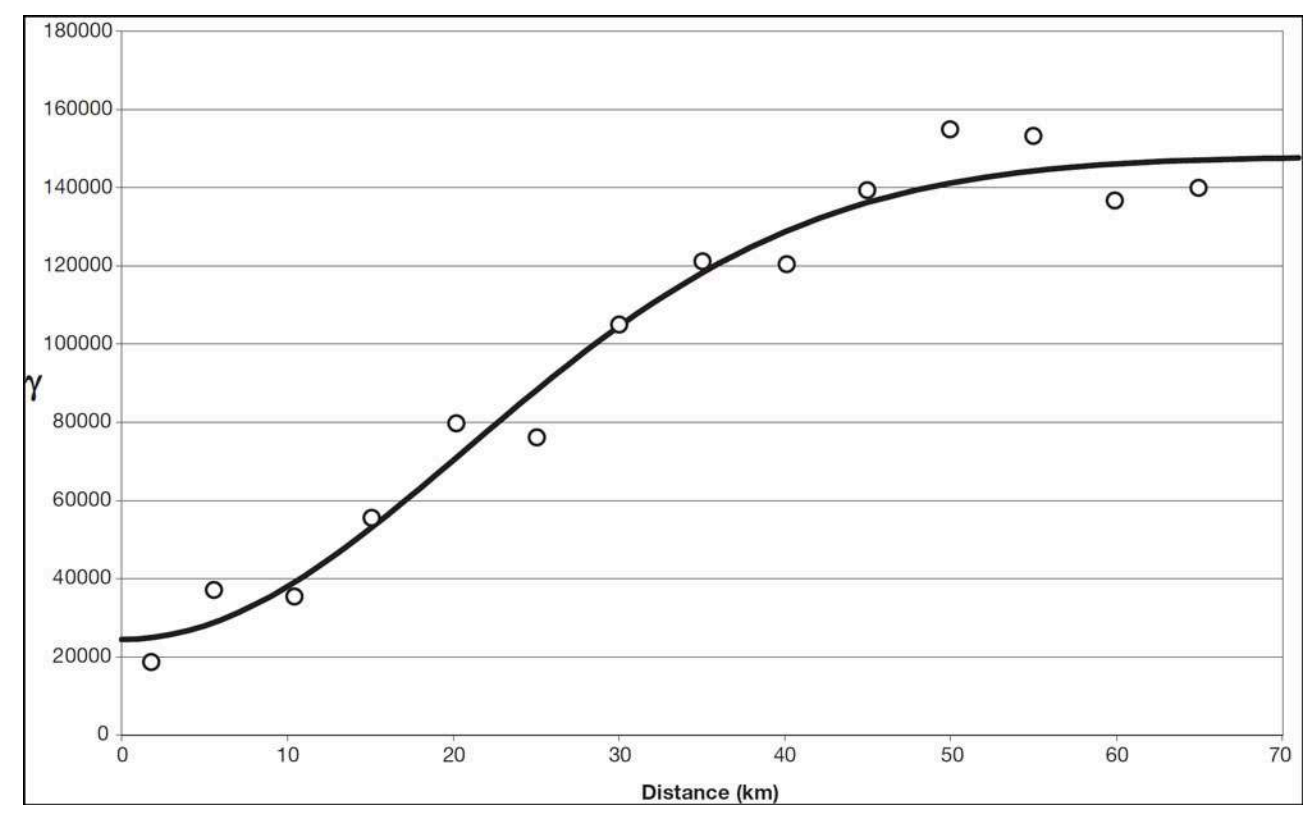

- Dans le domaine occidental, le Massif Ardennais au nord-ouest ainsi que l'Argonne et le Barrois à l'ouest constituent les régions les plus arrosées (respectivement 1300 et 1 $100 \mathrm{~mm}$ ). Une partie de la Champagne à l'extrémité nord-ouest et l'aval du bassin de la Moselle connaissent les hauteurs annuelles de précipitations les plus faibles, inférieures à $800 \mathrm{~mm}$.

- Dans le domaine oriental, les reliefs vosgiens déterminent des contrastes pluviométriques beaucoup plus importants. Le massif est constitué de 2 môles séparés par le bassin de Saint-Dié. Au Sud sur les Hautes Vosges et la Vôge, les précipitations dépassent régulièrement $2000 \mathrm{~mm}$. Dans les Vosges du Nord, le maximum pluviométrique atteint $1600 \mathrm{~mm}$. De part et d'autre du massif, les isohyètes se répartissent de manière dissymétrique. Le versant lorrain apparaît comme un glacis régulier alors qu'à l'est, le versant alsacien se caractérise par une diminution très rapide des précipitations qui n'excèdent pas $700 \mathrm{~mm}$ dans la Plaine d'Alsace.

Dans le détail, le tracé des isohyètes diffère de manière sensible. Pour la méthode du krigeage, la carte dépend intégralement de la répartition du réseau pluviométrique car l'altitude n'est pas utilisée ici en tant que variable auxiliaire comme en co-krigeage (Pardo-Iguzquiza, 1998). Le modèle de variogramme et les options choisies déterminent des isolignes de forme concentrique autour des stations. La méthode PLUVIA propose une carte où apparaissent tous les reliefs significatifs. Ainsi des isohyètes soulignent, de manière précise, les côtes de Meuse, de Moselle et les différentes arêtes du massif vosgien. Cette carte rend compte des régions qui subissent un effet d'abri au pied de ces mêmes reliefs comme dans la Woëvre, la vallée de la Moselle aval et la plaine d'Alsace. Sur le secteur oriental, elle présente d'ailleurs de fortes analogies de détail avec la carte 
figurant dans l'atlas du projet REKLIP concernant le Fossé Rhénan méridional et ses bordures (Fiedler et al., 1995), réalisée par la méthode AURELHY sur la période 1951-1980.

Ainsi ponctuellement, les écarts entre les valeurs du modèle de quantification et celles issues de la méthode du krigeage peuvent être relativement élevés notamment pour ces régions d'abri. Sur le versant alsacien des Vosges, des valeurs supérieures à $40 \%$ apparaissent. À l'ouest, sur le Plateau Lorrain, les altitudes sont certes moins contrastées mais les écarts atteignent tout de même $20 \%$ au pied des Hauts de Meuse et dans la vallée de la Moselle aval. À l'inverse, sur les sommets (crêtes vosgiennes, revers de plateau), les écarts demeurent faibles en valeurs relatives car compris entre 10 et $20 \%$.

La comparaison des précipitations moyennes par bassins-versants obtenues par les deux méthodes montre que des différences importantes peuvent apparaître pour des bassins de taille réduite, inférieure à $100 \mathrm{~km} 2$. Celles-ci diminuent corrélativement à l'augmentation de la surface du bassin versant. Par exemple, pour le bassin de la Bruche à sa confluence avec l'Ill (soit une superficie de $720 \mathrm{~km} 2$ ) les deux méthodes proposent des valeurs différentes d'à peine $4 \%$ alors qu'à l'intérieur de ce même bassin, les écarts peuvent être beaucoup plus élevés (35\%) pour des portions inférieures à 100 $\mathrm{km} 2$.

Outre le fait que la quasi-totalité des postes ait été utilisée, il convient de noter que, de toute manière, les stations disponibles font le plus souvent défaut dans les zones où les résultats des deux méthodes divergent. Une validation fiable de l'une ou l'autre approche nécessite donc une refonte complète des calculs, en isolant un lot de validation conséquent et judicieusement choisi.

Toutes les cartes issues de l'application de la méthode PLUVIA aux précipitations annuelles de la période 1971-1990 ne peuvent pas figurer dans cet article. Cependant, des cartes représentant les précipitations fréquentielles décennales sèches et humides rendent compte de la répartition spatiale des valeurs extrêmes annuelles (fig. 6a et 6b). Les précipitations annuelles de la région s'ajustent de manière satisfaisante à la loi normale. De la sorte, en connaissant les précipitations annuelles de 1971 à 1990 en chaque point de grille, l'application systématique de la loi normale sur les 20 valeurs permet d'estimer sur l'ensemble du maillage, la pluviosité relative aux fréquences précitées. Les cartes correspondantes reproduisent la configuration générale déjà constatée à l'échelle interannuelle. Cependant, il est intéressant de constater que la région peut connaitre, pour une durée de retour de 10 ans, des valeurs de précipitations extrêmement basses, inférieures à $500 \mathrm{~mm}$ dans la Woëvre et pour une large part dans la Plaine d'Alsace. En revanche, les principaux massifs, Vosges et Ardennes, demeurent toujours très arrosés. En situation décennale humide, les sommets des Hautes-Vosges enregistrent des hauteurs annuelles supérieures à 3000 $\mathrm{mm}$ alors qu'un peu plus à l'est, dans la région de Colmar, elles ne dépassent pas 600 $\mathrm{mm}$. Cette région apparaît donc comme particulièrement sèche et la pluviométrie annuelle y varie très peu comparativement à ce qui est observé dans la Woëvre.

Fig. 6 : Quantification Spatiale des Précipitations. Période de référence 1971-1990. 
6a : Précipitations annuelles décennales sèches

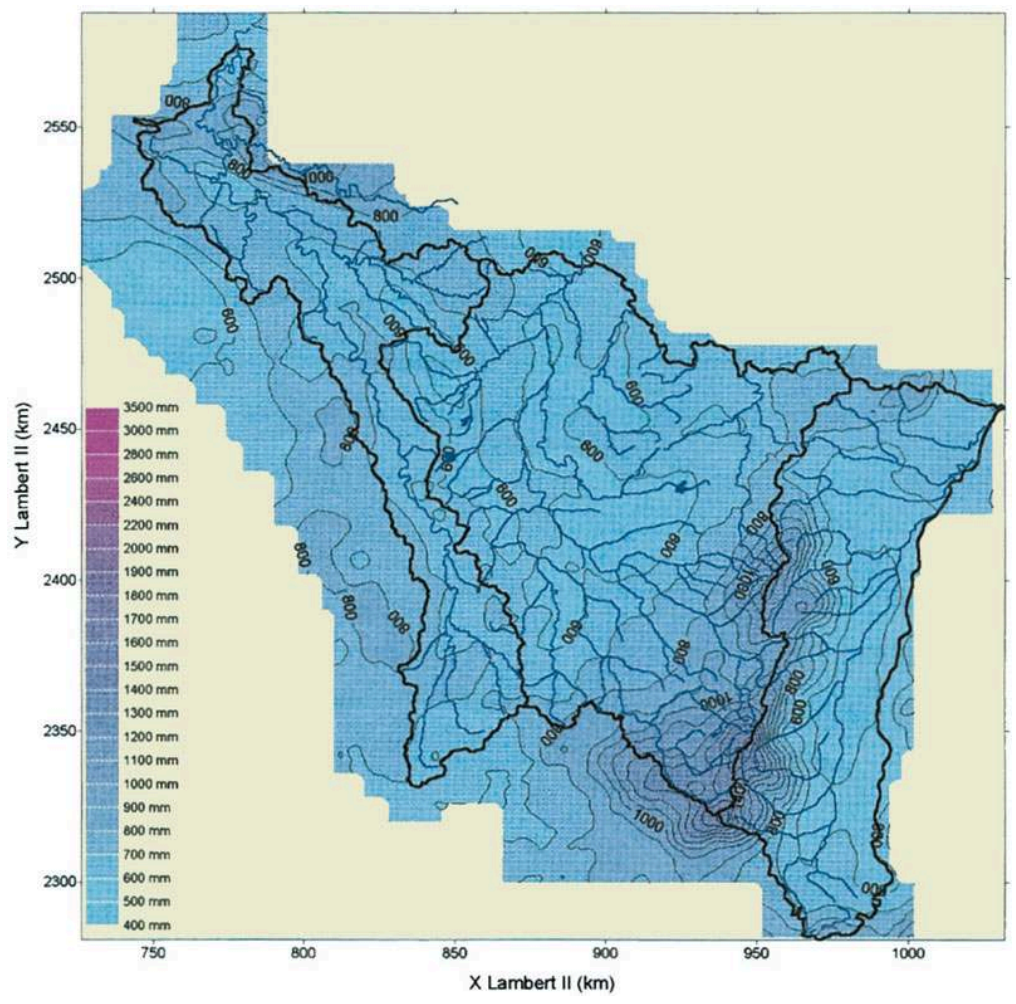

$6 b$ : Précipitations annuelles décennales humides

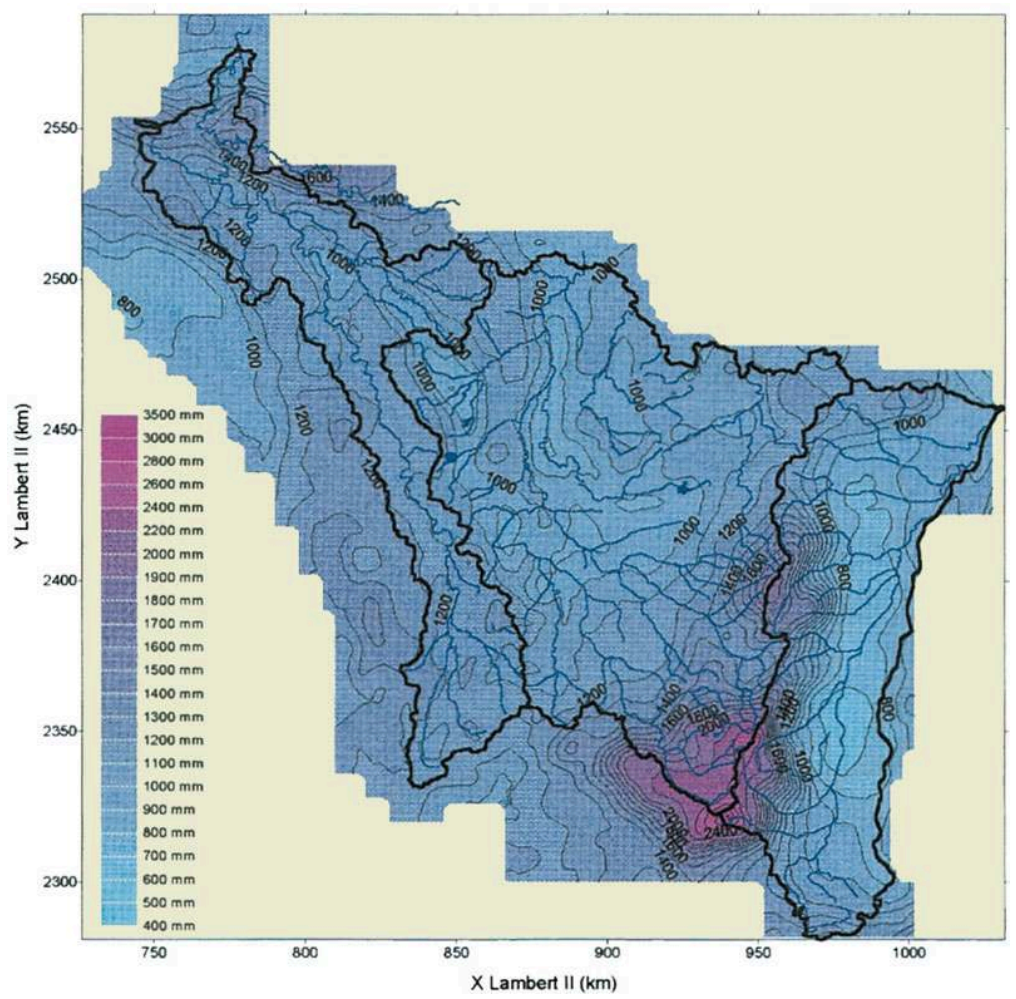




\section{Conclusion}

Relativement lourde à mettre en œuvre, la méthode mise au point par le CEREG a dû être automatisée. Grâce au principe de paramétrisation omnidirectionnelle, elle présente un réel intérêt pour la cartographie des champs de précipitations et l'estimation des précipitations moyennes par bassin versant. En combinant des paramètres locaux à des caractéristiques d'envergure régionale, elle fournit des résultats très satisfaisants pour des échelles spatiales relativement fines (petits bassins inférieurs à $100 \mathrm{~km} 2$ ). Mais par ailleurs, les protocoles de comparaison avec les méthodes géostatistiques doivent encore être affinés.

Son application à la France du Nord-Est a mis en lumière les reliefs expliquant le mieux la répartition des précipitations à l'échelle annuelle, tout en montrant que leur influence varie en fonction des types de temps. Dans certains cas, quelques effets générés par le modèle (effets d'abri locaux, par exemple) ne sont pas vérifiables, par manque de stations dans les secteurs concernés. Il y a là des points d'intérêt qui méritent d'être approfondis, afin de contrôler davantage la robustesse de l'approche.

Par ailleurs, des tests au niveau mensuel ont révélé des performances globalement satisfaisantes. L'exploitation des résultats et leur confrontation aux lames d'eau écoulées aux stations hydrométriques introduisent à présent d'autres recherches liées aux déficits d'écoulement et au bilan hydrologique.

\section{BIBLIOGRAPHIE}

Benichou P., Le Breton O. (1987). - Prise en compte de la topographie pour la cartographie des champs pluviométriques statistiques. La Météorologie, $7 \mathrm{e}$ série, $\mathrm{n}^{\circ} 19$.

Delhomme J.-P. (1978). - Kriging in the hydrosciences. Advances in Water Resources, 1 (5), pp. 251-266.

Englund E., Sparks A. (1991). - Geostatistical Environmental Assessment Software. User's guide. United States Environmental Protection Agency, Environmental monitoring systems laboratory, Las Vegas Nevada.

Fiedler F. et al. (1995). - Atlas climatique du Fossé Rhénan méridional. Projet REKLIP. Éditions Coprur, Strasbourg.

François D. (1996). - Quantification spatiale des précipitations du bassin Rhin-Meuse. Secteur occidental. 1re phase : Précipitations interannuelles de la période de référence 1971-1990. Rapport Université de Metz, CEGUM - Agence de l'Eau Rhin-Meuse.

François D. (1997). - Quantification spatiale des précipitations du bassin Rhin-Meuse. Secteur occidental. 2e phase : Précipitations annuelles de la période de référence 1971-1990. Rapport Université de Metz, CEGUM - Agence de l'Eau Rhin-Meuse.

François D., Gille E., Zumstein J-F. (1995). - Définition de régions pluviométriques dans les bassins de la Meuse, de la Moselle et des affluents alsaciens du Rhin. Rapport Université de Metz, CEGUM - Agence de l'Eau Rhin-Meuse. 
Humbert J., Perrin J-L., Perron L. (1993). - Étude méthodologique de quantification spatiale des précipitations appliquées à la France du Nord-Est. Secteur-test : Versant oriental des Vosges. Rapport CEREG/ULP Strasbourg - Agence de l'Eau Rhin-Meuse.

Humbert J., Perrin J.L. (1993). - Précipitations et relief. Le cas du versant oriental des HautesVosges. In L'Eau, la Terre et les Hommes, Presses Universitaires de Nancy, pp. 147-154.

Humbert J. (1995). - Cartographie automatique des précipitations mensuelles et annuelles en zone montagneuse. Annales de Géographie (581/582), pp. 168-173.

Humbert J., Mahr N., Siefert N. (1997). - Quantification spatiale des précipitations du bassin RhinMeuse. Secteur oriental. Période 1971-1990. Rapport CEREG/ULP Strasbourg - Agence de l'Eau RhinMeuse.

Humbert J., Perron L., Perrin J.L. (1997). - Precipitation mapping in mountainous area. Comparison of two statistical models. Proceedings of International Conference « Developments in Hydrology of Mountainous areas ». FRIEND-AMHY- IHP V, High Tatras, Slovakia, Technical Documents in Hydrology $n^{\circ}$ 8, UNESCO, pp. 70-75.

Humbert J., Drogue G., Mahr N. (1999). - Cartographie des précipitations par paramètrisation omnidirectionnelle du relief : la méthode Pluvia. In Actes 11e Colloque de l'Association Internationale de Climatologie (AIC), Lille, sept. 1998, à paraître.

Laborde J.-P. (1984). - Analyse des données et cartographie automatique en hydrologie ; éléments d'hydrologie lorraine. Thèse de doctorat, Université de Nancy, 484 p.

Mahr N., Humbert J. (1996). - PLUVIA : logiciel d'aide à la gestion et à la spatialisation des données pluviométriques à partir d'un MNT (WINDOWS/WINDEV), CEREG, ULP Strasbourg.

Marand C., Zumstein J.-F. (1990). - La modélisation des précipitations moyennes annuelles appliquée au Massif Vosgien. Hydrologie continentale, $n^{\circ} \quad 5 / 1$, pp. 29-39.

Obled C. (1987). - Introduction au krigeage à l'usage des hydrologues. 2es journées hydrologiques de l'ORSTOM, ORSTOM Collection Colloques et Séminaires, pp. 174-222.

Pardo-Iguzquiza E. (1998). - Comparison of geostatistical methods for estimating the areal average climatological rainfall mean using data on precipitation and topography ". International Journal of Climatology, 18, pp. 1031-1047.

Paul P. (1992). - Classification des situations météorologiques sur le Nord-Est de la France ; période 1981-1990. Rapport interne, CEREG, ULP Strasbourg.

Scherer J.C. (1977). - Une méthode d'extrapolation dans l'espace de données pluviométriques moyennes. Application à une partie des Vosges. Recherches Géographiques à Strasbourg (4), pp. 69-85.

\section{RÉSUMÉS}

Une méthode de spatialisation des précipitations (PLUVIA) a été appliquée aux précipitations annuelles de la période 1971-1990 de deux régions du Nord-Est de la France. PLUVIA a permis de déterminer les reliefs qui expliquent le mieux la répartition des précipitations annuelles. Les résultats ont également été comparés à ceux issus d'un krigeage simple pour estimer le gain de précision obtenu par PLUVIA.

A method of rainfall mapping (PLUVIA) has been applied to the annual rainfall of the period 1971-1990 of two regions of the Northeast of France. PLUVIA permitted the determination of 
reliefs that explain best the annual rainfall distribution. The results were also compared to those derived from an ordinary krigging in order to assess the increase in precision gained by PLUVIA.

Eine Methode zur räumlichen Erfassung der Niederschläge (PLUVIA) wurde auf die Jahresniederschläge der Periode 1971-1990 in zwei Regionen Nordostfrankreichs angewandt. PLUVIA ermöglicht es, die Ursachen zu bestimmen, die am besten die Verteilung der Jahresniederschläge erklären. Die Ergebnisse wurden zugleich verglichen mit denen eines normalen Krigging, um den durch PLUVIA erzielten Präzisionsgewinn abschätzen zu können.

INDEX

Schlüsselwörter : Modell, Niederschläge, Nordostfrankreich, räumliche Erfassung

Keywords : mapping, model, Northeast of France, rainfall

Mots-clés : modèle, Nord-Est de la France, précipitations, spatialisation

\author{
AUTEURS \\ DIDIER FRANÇOIS \\ CEGUM - Université de Metz - Ile du Saulcy - 57045 Metz Cedex 1
}

JOËL HUMBERT

CEREG (EP 2037 CNRS) - Université Louis Pasteur - 3, rue de l'Argonne 67083 Strasbourg Cedex 